\title{
A TNF-alpha Receptor Antagonist Etanercept and it's Role in The Treatment of Early Period of Sepsis \\ Kadiroğlu AK*
}

Department of Nephrology and Internal Medicine, Medicine Faculty in Dicle University, Turkey

\section{Letter to the Editor}

Sepsis is defined as the result of a systemic inflammatory response caused by infection causing microorganisms. The most common causes are severe pneumonia urinary tract infections, and intra-abdominal infections, such as peritonitis and bacteremia, induced by indwelling catheters, often leads to multi-organ dysfunction and the kidney is one of the organs frequently afflicted. It is still a major cause of morbidity and mortality in the intensive care unit. Acute kidney injury (AKI) occurs in about $19 \%$ patients with moderate sepsis, $23 \%$ with severe sepsis and 51\% with septic shock, when blood cultures are positive. Upon bacterial infection or exposure to a large range of microbial products, two major types of systemic dysfunctions may be the basis of sepsis. On the one hand, an overwhelming inflammation may cause a profound suppression of the immune response. On the other hand, sepsis may induce a powerful and devastating immune reaction leading to excessive production of inflammatory mediators which, by inducing fever, cardiovascular dysfunction, and multiple organ failure especially acute kidney injury, are harmful for the host.

In fact, proinflammatory cytokines such as TNF- $\alpha$ and IL-1 are increased as a essential part of the inflammatory response in order to limit tissue damage. However, overproduction of these proinflammatory cytokines disrupts the normal immune response and causes a pathological response. As a result, capillary leakage, tissue damage, and multiorgan failure especially acute kidney injury can occur. TNF- $\alpha$ plays a central and an important role in this process and is considered as the master mediator in the early stage of inflammation which was secreted minutes after the inflammatory stimulus, peaked at first hour, and stopped after 3-4 h. It was revealed that in vivo injection of TNF- $\alpha$ partially recur symptoms of septic shock by inducing hypotension, cardiac dysfunction, and vascular leakage. Hence it is essential and important to develop new therapeutic strategies against to the TNF- $\alpha$.

Antibiotics have great importance and play a main role in the treatment of inflammation and sepsis. Unfortunately, in the many clinics antibiotics cannot start in time and so the treatment of sepsis can be inadequate. However, many researchers have compared the effectiveness of various antibiotics in sepsis, but in clinical practice they have not obtained any eligible results. Therefore, there is a need for new therapeutic strategies in the treatment of early period of sepsis.

Etanercept is a soluble fusion protein and is the competitive inhibitor of TNF- $\alpha$ which inhibits the binding of TNF- $\alpha$ to cell surface receptors which are expressed also in the glomeruli, limits its biological activity. Suppression of TNF- $\alpha$ by etanercept in the case of excessive immune response can play an important role in the limiting of inflammation. It is approved for subcutaneous use in the treatment of patients with moderate to severe active rheumatoid arthritis, juvenile rheumatoid arthritis, psoriatic arthritis, ankylosing arthritis and plaque psoriasis in the United State, Italy, the rest of the European and other countries worldwide. It was also shown that etanercept can improve neuroinflammation and myocardial ischemia reperfusion injury. The successful results obtained from the use of Etanercept in the inflammatory disease of the joints, neuroinflammation and myocardial ischemia/reperfusion injury are encouraging.

Consequently, etanercept, in addition to antibiotics given in the treatment of early period of sepsis, results in more significant improvement in inflammatory parameters and process according to antibiotics alone. I believe that etanercept can decrease sepsis incidence and multiorgan failure such as acute kidney injury but there is a need for more comprehensive experimental and clinical studies for usage in the treatment of early period sepsis.
*Corresponding author: Prof. Dr. Ali Kemal Kadiroğlu, Department of Nephrology, Medicine Faculty in Dicle University, Diyarbakir, Turkey, E-mail: akkadiroglu63@gmail.com

Received: April 11, 2016; Accepted: April 15, 2016; Published: April 20, 2016

Citation: Kadiroğlu AK (2016) A TNF-Alpha Receptor Antagonist Etanercept and it's Role in The Treatment of Early Period of Sepsis. J Kidney 2: 123. doi:10.4172/2472-1220.1000123

Copyright: (๑) 2016 Kadiroğlu AK. This is an open-access article distributed under the terms of the Creative Commons Attribution License, which permits unrestricted use, distribution, and reproduction in any medium, provided the original author and source are credited. 